Supporting information of "Theoretical Studies on the Photochemistry

of Pentose Amino-oxazoline, a Hypothetical Intermediate Product in

Prebiotic Synthetic Scenario of RNA Nucleotides"

Yuejie $\mathrm{Ai}^{a}{ }^{a}$, Shuhua Xia ${ }^{b}$, Rongzhen Liao ${ }^{c, *}$

${ }^{a}$ School of Environmental and Chemical Engineering, North China Electric Power University, Beijing 102206, China

${ }^{b}$ College of Life and Environmental Science, Minzu University of China, Beijing100081, China

${ }^{c}$ Key Laboratory of Material Chemistry for Energy Conversion and Storage, Ministry of Education, Hubei Key Laboratory of Materials Chemistry and Service Failure, School of Chemistry and Chemical Engineering, Huazhong University of Science and Technology, Wuhan 430074, China

E-mail: aiyuejie314@126.com rongzhen@hust.edu.cn

Table S1. Vertical excitation energies in eV (nm), oscillator strengths (f) for the low-lying singlet excited states of the pentose amino-oxazoline and its protonated forms at the MS-CASPT2//CASSCF(8,8)/6-31G(d) level of theory.

\begin{tabular}{ccc}
\hline state & $\Delta \mathrm{E}, \mathrm{eV}(\mathrm{nm})$ & $f$ \\
\hline Neutral model & & 0.0232 \\
$\mathrm{~S}_{1}\left(\pi \pi^{*}\right)$ & $7.32(169)$ & 0.1262 \\
$\mathrm{~S}_{2}\left(\mathrm{n} \pi^{*}\right)$ & $7.45(166)$ & \\
Protonated model & & 0.6768 \\
$\mathrm{~S}_{1}\left(\pi \pi^{*}\right)$ & $7.62(163)$ & 0.0063 \\
$\mathrm{~S}_{2}\left(\mathrm{n} \sigma^{*}\right)$ & $8.94(139)$ & \\
Neutral water model & & 0.0302 \\
$\mathrm{~S}_{1}\left(\pi \pi^{*}\right)$ & $7.19(172)$ & 0.6789 \\
$\mathrm{~S}_{2}\left(\mathrm{n} \pi^{*}\right)$ & $7.59(163)$ & \\
$\mathrm{S}_{1}\left(\pi \pi^{*}\right)$ & & 0.6917 \\
$\mathrm{~S}_{2}\left(\mathrm{n} \sigma^{*}\right)$ & $7.72(160)$ & 0.0045 \\
\hline
\end{tabular}


Figure S1. Transition molecular orbitals for low-lying excited states of the pentose amino-oxazoline in vacuum.

\section{Neutral model}

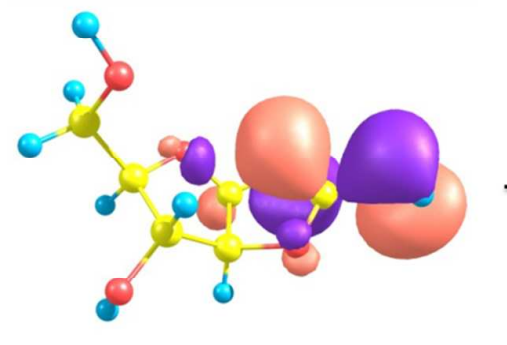

MO-46

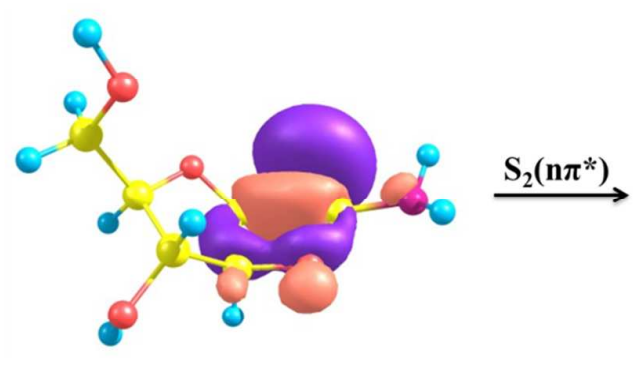

MO-44

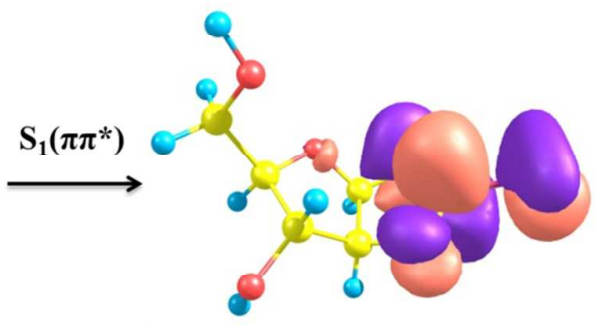

MO-48

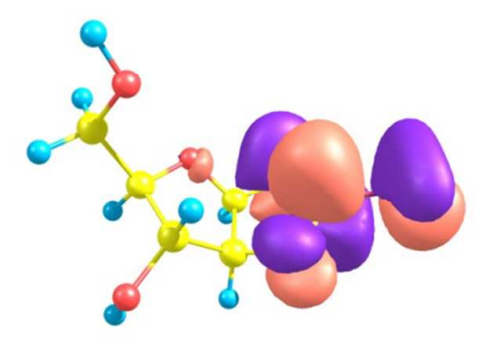

MO-48

\section{The Linear interpolations in internal coordinates (LIIC) calculations}

The multistate complete active-space second-order perturbation (MS-CASPT2) approach is exploited to evaluate the energies of linearly interpolated internal coordinate paths. An active space of 8 electrons in 8 molecular orbitals and 6-31G* basis set is employed for the MS-CASPT2 computations. The polarizable continuum model with water solvent was used as implicit water model. All MS-CASPT2 calculations are performed using MOLCAS8.0. 


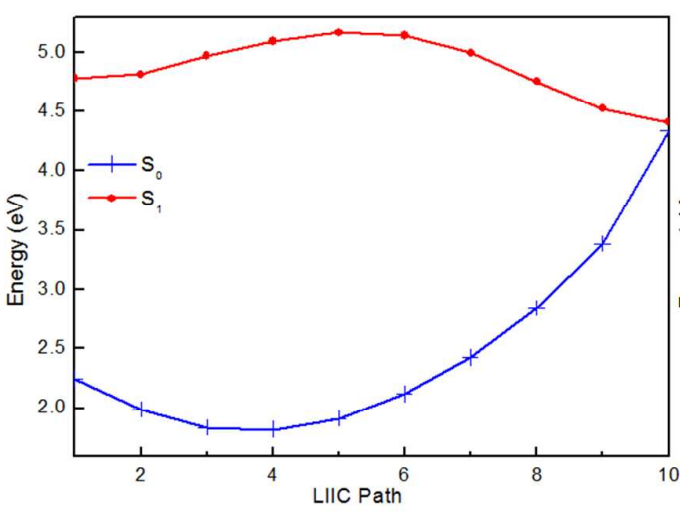

GAS $(8.9 \mathrm{kcal} / \mathrm{mol})$

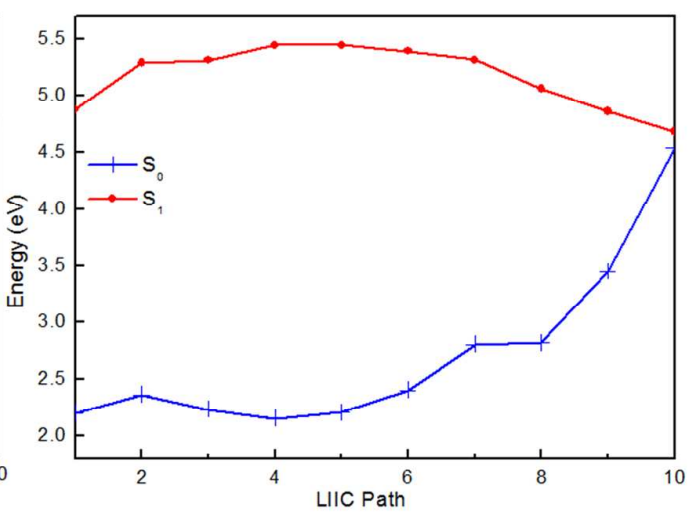

PCM (13.1kcal/mol)

(a)

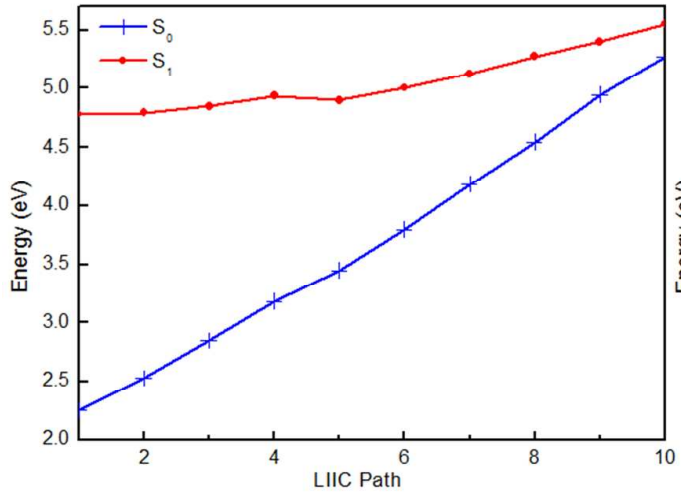

GAS (17.8kcal/mol)

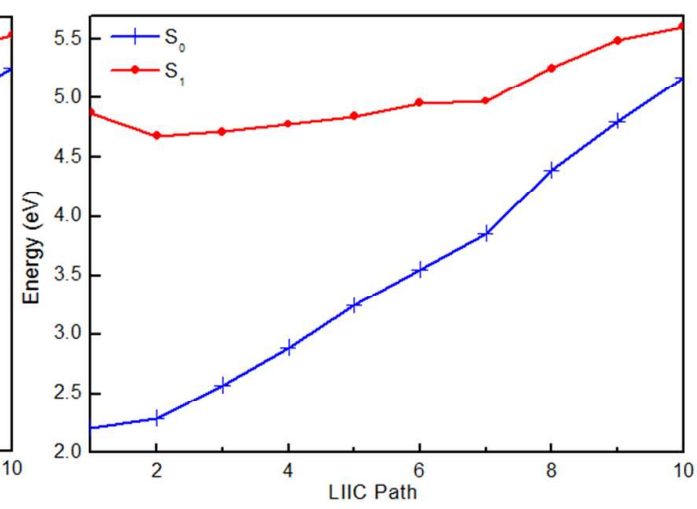

PCM (16.8kcal/mol)

(b)

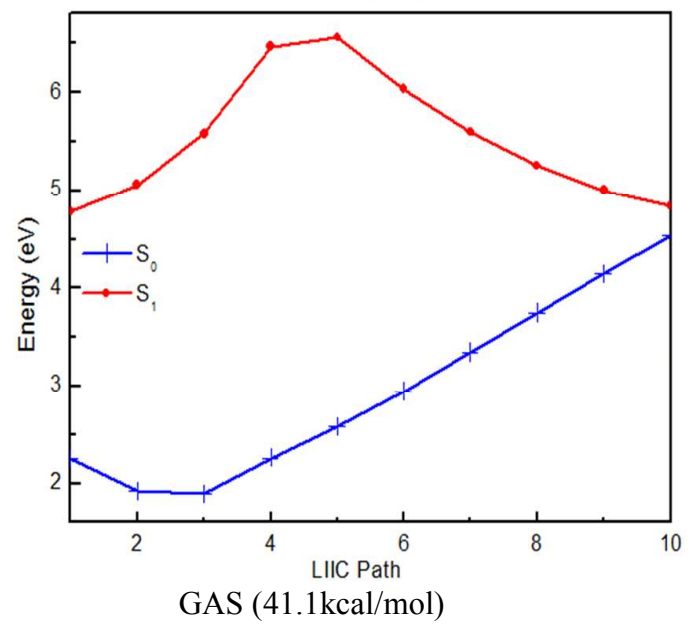

(c)

Figure S2. MS-CASPT2 computed linearly interpolated internal coordinate (LIIC) paths connecting the $\mathrm{S}_{1}$ minima and the (a)CI-bend, (b) CI-CN and (c) CI-NH 
structure in gas phase (left) and using PCM model (right).

Figure S3. Transition molecular orbitals for low-lying excited states of the protonated pentose amino-oxazoline in vacuum.

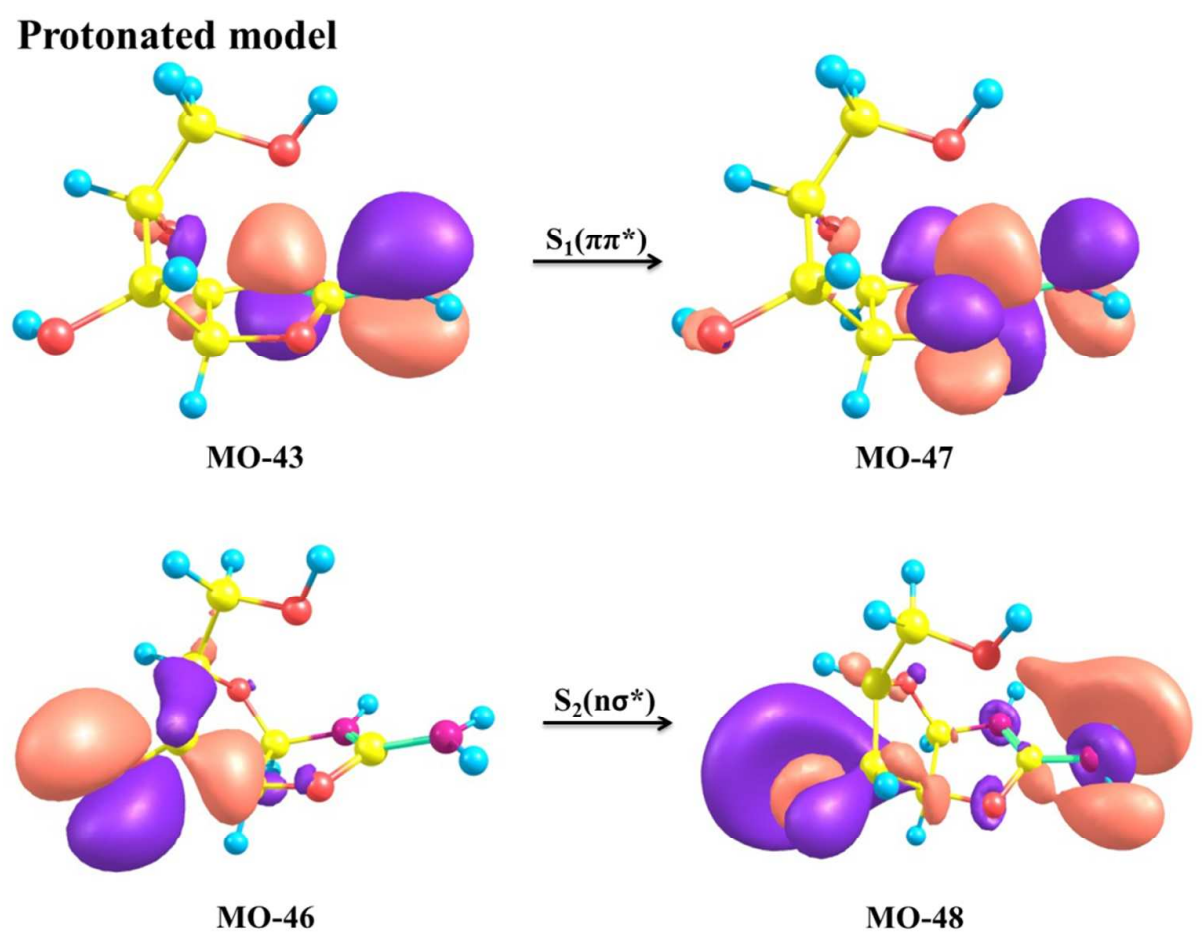

Figure S4. The optimized structure for CI-N18H23 of protonated pentose amino-oxazoline, calculated at $\mathrm{CAS}(8,8) / 6-31 \mathrm{G}(\mathrm{d})$ level.

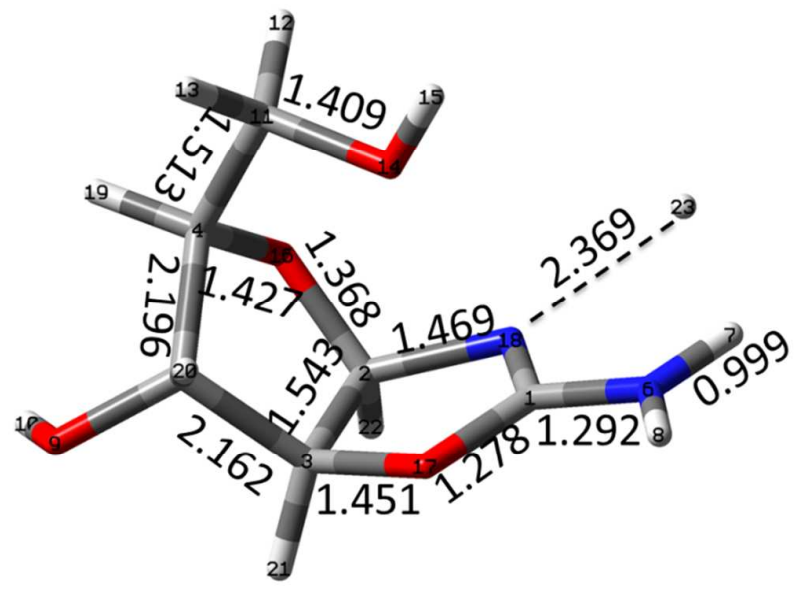

\section{CI-N18H23}

Figure S5. Potential-energy profiles along different bond coordinates in the ground state $S_{0}$ and the first excited state $S_{1}$ for the neutral molecule in the solution. The 
profiles were obtained by a rigid scan at the TDDFT/PCM/B3LYP/6-31G(d) level.
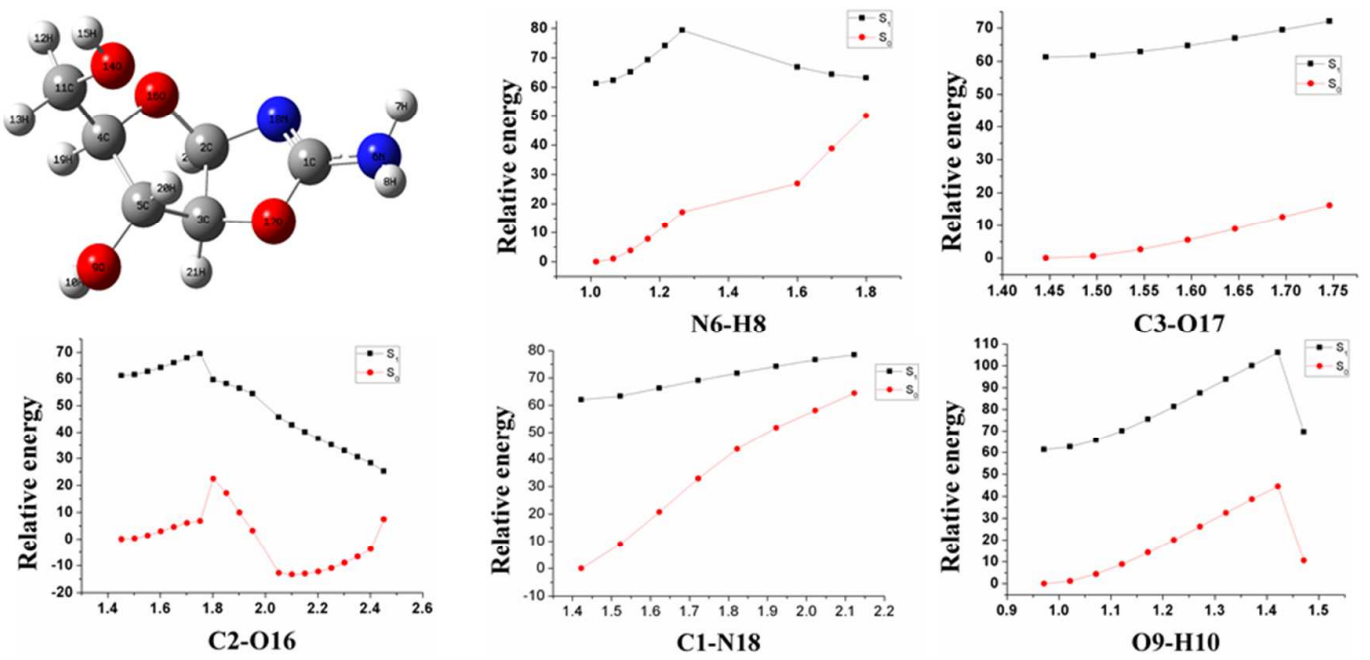

Figures S6. Potential-energy profiles along different bond coordinates in the ground state $S_{0}$ and the first excited state $S_{1}$ for the protonated molecule in the solution. The profiles were obtained by a rigid scan at the TDDFT//PCM/B3LYP/6-31G(d) level.
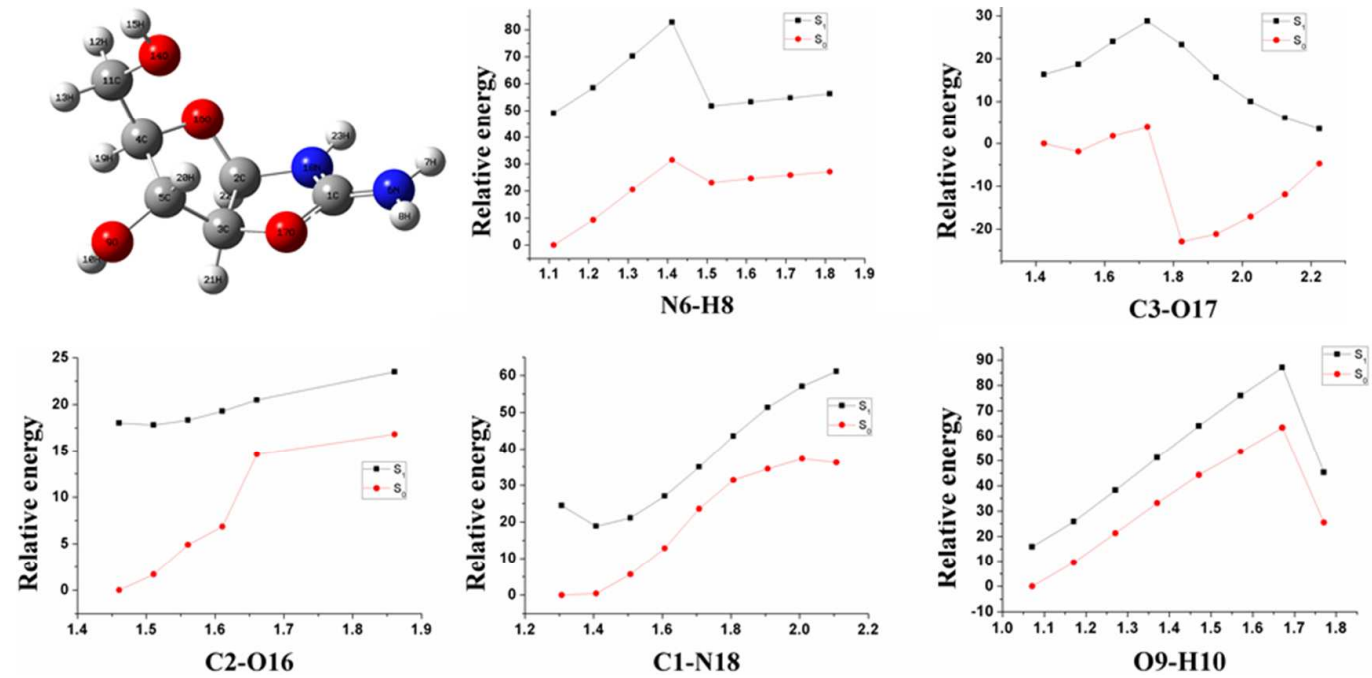
Figure S7. Transition molecular orbitals for low-lying excited states of the pentose amino-oxazoline in water solution.

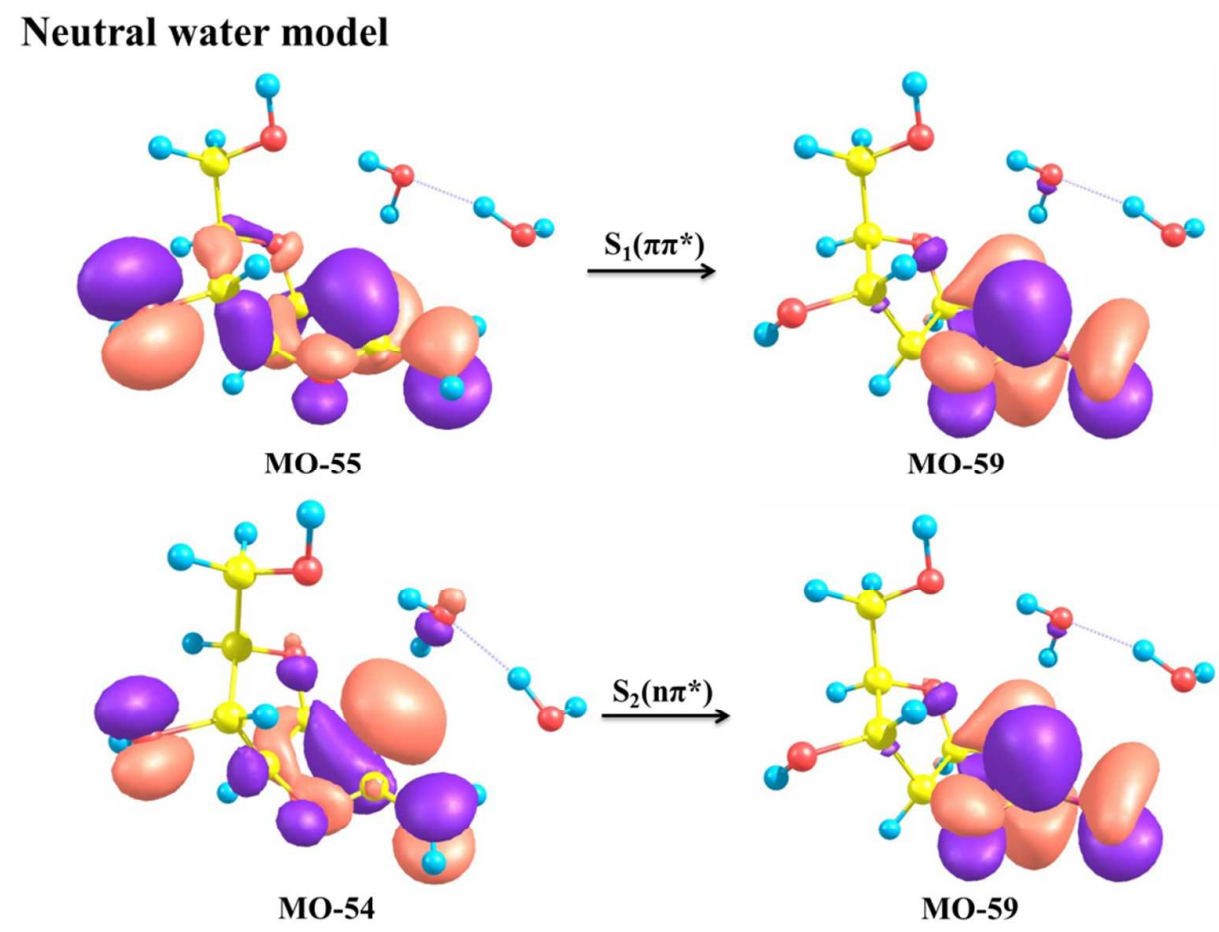

Figure S8. Transition molecular orbitals for low-lying excited states of the protonated pentose amino-oxazoline in water solution.

\section{Protonated water model}
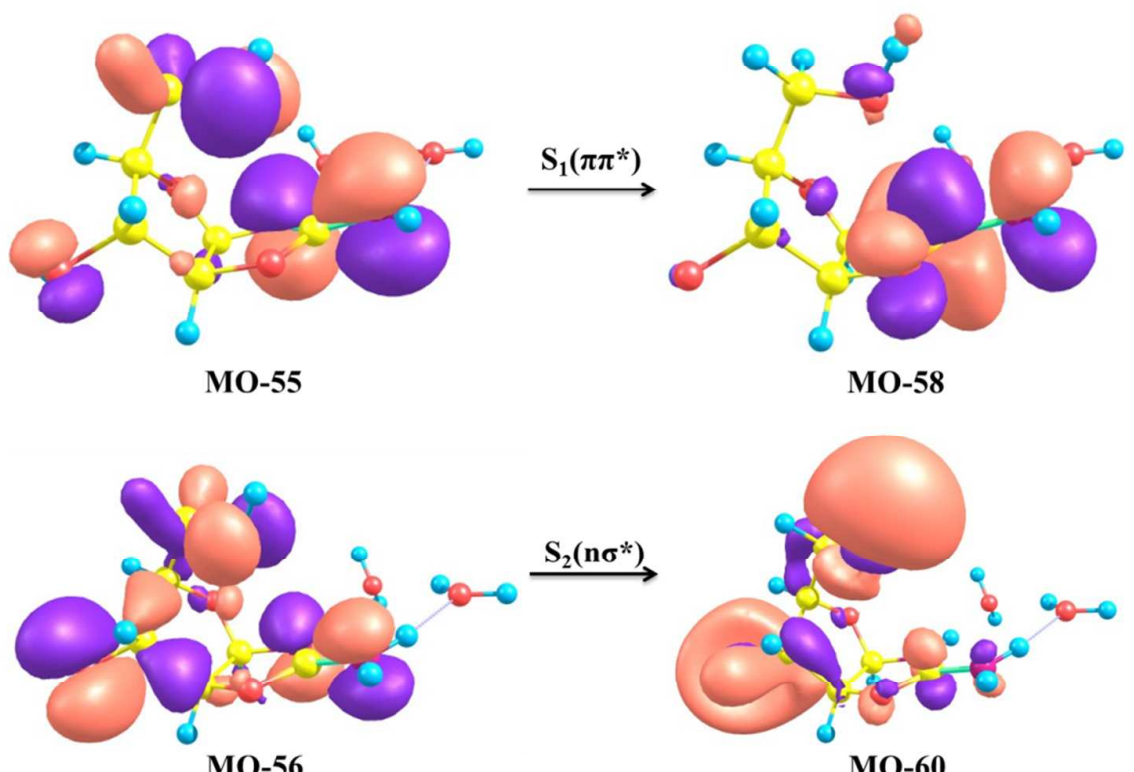
Table S2. CASPT2//CAS(8,8)/6-31G(d) single point energies at the CI structures on top of the optimized structures at the $\mathrm{CAS}(8,8) / 6-31 \mathrm{G}(\mathrm{d})$ level in vacuum.

\begin{tabular}{ccccc}
\hline & \multicolumn{2}{c}{ neutral } & \multicolumn{2}{c}{ protonated } \\
\hline & \multicolumn{2}{c}{ CASPT2 } & $\mathrm{S}_{0}$ & $\mathrm{~S}_{1}$ \\
& $\mathrm{~S}_{0}$ & $\mathrm{~S}_{1}$ & -643.3461224 & -643.3323988 \\
CI-NH & -642.9696469 & -642.9584419 & -6459 & -643.3349146 \\
CI-CN & -642.9427301 & -642.9322939 & -643.3445656 & -6436 \\
CI-bend & -642.9767463 & -642.9742832 & -643.3772869 & -643.3613609 \\
\hline
\end{tabular}

